\title{
Ontology Construction of Online Education
}

\author{
Xiaoguang $\mathrm{Zhu}^{1, *}$, Fengzhao Chen ${ }^{1}$ and Yu Zhang ${ }^{1}$ \\ ${ }^{1}$ School of Management Engineering, Xuzhou University of Technology, Xuzhou, Jiangsu 221018, China \\ *Corresponding author. Email: zhu_xg@xzit.edu.cn
}

\begin{abstract}
To describe novel patterns of online education, an education ontology is constructed and instantiated. Current education ontology focused on traditional education resources and procedures, and lacked specification of online education. An education ontology was induced and codified to describe online courses, activities, platforms and discussion forum with Protége software. This is the first time to construct ontology of online education with multiple aspects. As a result, the constructed ontology uncovered the underlying patterns of online education and commonality among online education platforms. The patterns could support the integration of online education resources and promote innovation of online education strategy.

Keywords: ontology construction; online education; learning activity; education resource; platform
\end{abstract}

\section{INTRODUCTION}

\subsection{Patterns of Online Education}

Information technology and data science have promoted creation and integration of education resources and teaching methods [1]. Many education models have been developed to adapt the technology and data analysis, such as online education, flipped classroom and blended learning [2]. Morden education models usually are compatible with cloud computing and platforms which are virtualizing the majority of educational activities, such as teaching, discussion and assessment. Students could watch lecture video and finish homework in an online environment. In advance, experiments and software could be performed with online compilers and computer-aided software.

There are three trends in those developments. Firstly, the scale of participants and resources are increasing quickly, hence require advanced data analysis to resolve education patterns. Secondly, roles and activities of participants are blended and becoming nonlinear. Online education could support multiple interactions simultaneously to elevate the flexibility of online learning procedures. Hence, students could start learning with any materials and procedures. Thirdly, education resources are hosted and distributed with support of knowledge bases and cloud services.

\subsection{Current Ontologies}

Current ontologies of education are focused on courses, schools, procedures and behaviours. Shema.org hosted types of educational organizations, events and courses [3]. For example, Properties of EducationEvent are assesses, educationalLevel and teaches; properties of event are actor, attendee, composer, etc. Those schemas have not discerned the special elements of online education. Recent studies developed specific ontology on educations. For example, an ontology-based framework is proposed to model goals, instructional processes and instructional material of the eLearning system [4]. University ontology defined educational activities and resources with classes, instances and retrieval methods [5]. Most axioms in those ontologies conformed organization and procedures of traditional educational institutions.

Domain knowledge and ontology technology have been applied to design curriculum and experiments. For example, linked data has been applied to map ontologies of education materials and labour knowledge bases [6]. Domain knowledge could specify teaching content but ignored education patterns and auxiliary teaching activities. In addition, many standards were developed to qualify and improve online educations, such as ISO/IEC 2382-36 and CELTS42. ISO/IEC 2382-36 provided terms and definition for vocabulary used in the field of learning education and training(LET), such as assessment, assistive technology and online learning. Those terms are common concepts that have not specified the resources and activities of online education. CELTS42 focused on the classification of fundamental education resources which has similar function to domain knowledge.

\subsection{Problem and methodology}

The problem is aiming incompleteness of current ontologies when describing objects and activities of online education. The incompleteness could hinder development of computational education, since specified schema is an antecedent to analyse and share educational resources. In another word, we need to update current ontologies to improve online education and support their data integration and analysis when developing novel computational education models. 
The study includes three steps. First, collect online education resources and activities through engagement of online courses. The engaged courses are hosted on platforms of edx.org, coursera.org, and ilab-x.com. Second, induce and specify novel schemas from those resources. Third, codify education ontology by protégé software, and add classes and instances from an online course PennX+BDE1x_Big Data and Education at edx.org.

\section{RATIONALE OF ONLINE EDUCATION}

Education patterns and information technologies are coevolving with equal positions. Mature schemas in each domain are not capable to dominate the development of online education. Knowledge exchange has to adapt information technologies, but the essence of knowledge exceeded substrates in cyberspace, such as the acquisition of implicit and operational knowledge. Hence, to support computational education model, we should analyse rationales and mechanisms of online education for construction of education ontology.

\subsection{Platform and Technology}

The driving forces of online education are virtualization approaches of education activities with support of cloud platforms, computation and statistical models. The platforms provided sufficient functions to visualize educational materials and support virtual communication, virtualization and human-machine interactions, such as map annotation, linked data and social network analysis. Morden information technologies are sufficient to support both static and dynamic services of online education. The static services are traditional information systems to support video, email and map services. The dynamic services are relatively advance to support cloud computing and virtual laboratory. They could facilitate acquisition of operational and implicit knowledge with combination of online education.

\subsection{Distributional Resources}

Information technologies promoted creation of educational resources. In the vast cloud platforms, education resources and private-provided goods are distributed with various types and accessibility. This distributional attribute is a reason to apply blended learning and flipped classroom. To adapt development of market and technology, education model should accommodate participant-provided contents as teaching materials.

The participant-provided contents have different qualities. To improve quality of online education, platforms were integrated and filtered the contents gradually. Except for traditional activities of learning, teaching and discussion, there is an underlying cue of those activities to sustain quality of online education. The technology could support online education, but could not distil knowledge by itself. Hence, learning activities of online education have been guided to improve quality of contents in education routines New content will be filtered and corrected by post rule, vote, peer assessment and lecturer approval. In the end, the guided learning activities will improve quality of online education resources.

\subsection{Educational Activities}

In online education platforms, activities are correlated to cognitive, psychological and social aspects. An action usually could not be interpreted to a specific aspect, since their underlying motivations are mixed. Behaviour analysis showed that participants are motivated by various factors, such as pleasure, achievement and self-presentation. Those factors also correlated to personal characteristics.

To motivate participant with the differentiation, platform guidance has been designed to promote engagement and retention of participants. Common methods are feedback, interaction and goal orientation. Related studies have validated the utilities of this feedback, active learning, visualization and peer assessment. Correspond to this conclusion, platforms have provided optional actions to motivate participants in the learning process.

\section{NEW ONTOLOGY}

Online educations are developing novel patterns on many aspects corresponding to advance technologies. The prominent technologies are cloud computing and multimodal interactions. Through the various interactions, students could provide educational materials and exchange knowledge like a lecture. With increased scales of students, online education materials and activities will be diversified toward multiple aspects.

Table 1 presented differences between online education and traditional education in six aspects. It indicates that students have engaged in online education with more types of activities.

Table 1. Novel Patterns of online education

\begin{tabular}{|l|l|l|}
\hline Aspect & Traditional & Online \\
\hline Organization & Centric & Distributional \\
\hline Teaching & Linear & Multiple, specification \\
\hline Material & Uniform & Various, user provided \\
\hline Assessment & Batch & Instant, peer assessment \\
\hline Review & Lecturer & Peer, automation \\
\hline Discussion & Class, group & Discussion forum \\
\hline
\end{tabular}

In those differences, the prominent aspects are education material and grading assessment. Education materials have been expanded and diversified dramatically. Those materials could transform almost every learning activity from traditional classroom to online education platform, 
such as listen, discuss and complete homework. The platform also generalized the learning process. When participant provides materials, surveys or questions, they are already engaged in a learning procedure. When they assess question or assignment of other participants, they could also accomplish the learning procedure and acquire knowledge with a specific type.

The educational resources usually have a distributional organization in current cyberspace. With the free boundary of cyberspace, social activities could easily be transferred from social platforms to education platforms. As many studies validated, online education platform implied cognitive, psychological and social activities. The teaching and review are prone to multiple roles oriented. With engagement to an online course, participants are free to play different roles in those processes.

\subsection{Classes of Objects}

Objects in the ontology are elements that may be confronted by participants. In the course presentations, the objects are DOM objects in online course sites. They have data types that conform to HTML language. But in the practice of education, they convey educational types of traditional instruments and virtualized materials. six classes of those objects are induced, as table 2 presented.

Table 2. Objects of Online Education

\begin{tabular}{|l|l|}
\hline L1 & L2 \\
\hline asset & assignment, lecture, stream, syllabus \\
\hline course & forum, lesson \\
\hline institution & company, university \\
\hline participant & instructor, mentor, student \\
\hline platform & compiler, coordinator, resources \\
\hline UGC & answer, comment, question, submission \\
\hline
\end{tabular}

The assets are materials published by course provider which could be university or company. A course usually has two or three lecturers in a single provider. The lecturer provides primary assets and may add references from external resources.

As same as the connotation of traditional education, the course is a series of lessons or lectures on a particular subject. It also included forum and lesson to adapt micro courses of online education. Compare to traditional education, the discussion forums are become more usual and important to facilitate knowledge exchange and motivate participants.

The platform is a type of computer system to provide services of collaboration and information processing. It is a critical point to exert potential of modern technology on online educations. UGC means user-generated content hosted by platforms as primary resources to improve education and motivate participants. Typical UGCs are threads in forum and submissions corresponding to an assignment.

\subsection{Classes of Properties}

Properties of objects are associations between classes of those objects. Since all objects of online education are virtual, this study only defines synthesized properties which are related to participant and instruments. That is, omitted formal association which belong to pure data analysis, such as text analysis and statistical model.

Compare to the objects, properties have a relatively clear boundary. Objects and motivations may not capable to be fully discerned, but the action of participants are specific as the platform designed. That is, online education platform has to define actions explicitly to guide the learning process, such as play video or subscribe a thread, but not necessary to explain a video or map. This study expanded the property to three levels, as table 3 presented.

Table 3. Activities of Online Education

\begin{tabular}{|l|l|l|}
\hline L1 & L2 & L3 \\
\hline Action & assessment & Assign, review, submit \\
\hline & Enroll & \\
\hline & Lesson & Learn, publish \\
\hline & Post & Quest, answer, subscribe, \\
\hline Relation & Belong_to & Institution, Instruction \\
\hline & Hosted_by & \\
\hline
\end{tabular}

The actions are associates with participants, such as lecturer, mentor and student. Generally, as users of the education platform, all participants could learn, discuss and assess toward an online course, except publishing asset which is limited on provider and lecturer of a course. In the beginning, a participant usually engages a course and learn their lessons. In the learning process, participants could submit homework to complete assignments and discuss them in forums.

The relations are associates with instruments, such as institution and instructor. All instruments and platforms have their properties, and most are compatible with each other, such as corporation of university and company to commence an education model or virtual laboratory, or combination of social and education platforms. In addition, the objects could be re-organized flexibly, such as subforum could belong to lessons, or clustered under topics.

\subsection{Platforms}

As a critical point, course designer should discern types of relevant platforms. Students may learn a course in the integrated platform, such as Coursera, EDX, and perform a linear process. The education platform usually provided linear process and guidance to support continuity of learning, as buttons of previous and next indicated. The multi-modal information in online education has been designed to accommodate participant's habits. But when solve technique issues or integrate education resources, the course designer or data engineer should explore the 
underlying platforms, because they may cause different technique issues and convey different knowledge types.

Primary platforms are compiler, coordinator and resource. Compiler receives programs or other submissions, and return their scores with pre-defined measurements. It has two components: one is the compiler of domain knowledge; another is learning mechanisms about feedback and reinforcement. The coordinator will aggregate content provided by participants to support analysis and decision. Resource service is a typical information system to store, organize and retrieve information with vast compatibility and expression of hypermedia.

\section{RESULTS AND DISCUSSION}

Corresponding to the rationales and novelties, a primitive education ontology has been codified and hosted on GitHub [7]. In future, the ontology will be updated to describe more patterns of online educations, such as threads of discussion forum and procedures of novel education model, such as flipped classroom and virtual laboratory. The procedure could be described and illustrated from formal classes and typical instances by the education ontology.

The education ontology includes 78 logical axioms and 61 declaration axioms. Objects have 24 classes, and object property has 16 properties. The individuals are selected from the first two weeks of course PennX+BDE1x on EDX platform and annotated with descriptions and labels.

\subsection{Individuals}

This section illustrates classes with typical individuals. In the following figures, there are three edge types: 1) edge between classes is sub_class or property, 2) edge between class and individual is has_individual, 3) individuals related to each other with confirmation to their classes.

In figure 1, an assignment Assignment_Week_l has been hosted_by platform Assitments which is a compiler. A compiler could compile submissions of the assignment and return answers to grade student. The solution of the assignment may be assessed by peers or compiler with predefined criteria from a lecturer.

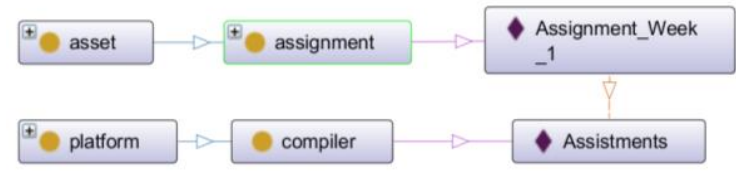

Figure 1. Individual of Compiler

In figure 2, instructor Ryan_Baker has published materials of intro_Video and The_State of Edu.... The 'publish' is a property between classes of instructor and asset. That means an instructor could publish an asset. The instructor affiliates institution of PennX, and provided the course Big_Data_and_Education.

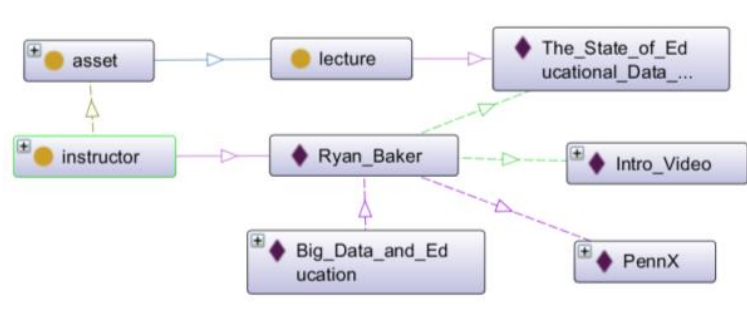

Figure 2. Individual of Instructor

An assignment may associate with lesson, material and platform. In figure 3, The Assignment_2 belong to an asset and hosted by the platform of resources. In addition, it also belongs to Week_l as a lesson and co-assigned with other materials of the lesson. Compare to other assets, assignment is more flexible to incorporate modern education strategies.

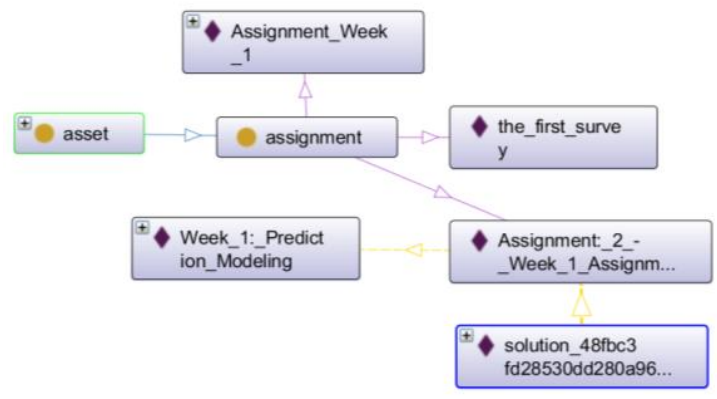

Figure 3. Individual of Assignment

The individuals of ontology are expressed in EDX platform with a unified organization. But in underlying, they have distributed storage and corroboration when exchange knowledge in an online environment. Education materials in the platform could be mapped to external linked data and knowledge bases. In addition, appraisal and assessment are usually accomplished through corroboration and a trusting network.

\subsection{Applications of Online Education Ontology}

The education ontology can describe various aspects of online education more precisely. Once education resources are described, they could be integrated and analysed by statistical models. Statistical analysis is a premise to promote computational education and decision making. Also, behaviour analysis could resort the ontology to achieve fast modelling of the cognitive, social and psychological activities. For underlying platforms, ontology also could depict issues caused by different platforms, such as technical issues and video accessibility. In addition, the ontology could support the structural optimization of a computational model. Firstly, it could support knowledge base population by bridging the online education resources and knowledge bases. Secondly, it could expand feature space of related statistical model or 
text analysis model, because the classes clarified the underlying platforms and education resources.

\subsection{Issues and Future Works}

The ontology is primitive and incomplete. In the future, we will add detailed classes to cover all kinds of assignments and discussions. In the process of completion, we will branch the ontology as well, since the entire ontology is too intricate, and a specific educational pattern will only use part of the ontology.

Based on the primitive ontology, we will apply statistical analysis to enhance the induction of education resources. For example, learning topics and activities could be resolved by content analysis models. In the end, we will resort to collaborative annotation and validation to enhance the cohesion and precision of the education ontology.

\section{CONCLUSION}

Information technology has promoted online education toward novel and diversified patterns. Without clear schemas, those patterns will hinder data analysis of online education resources. This study constructed an education ontology to depict online education with multiple aspects of objects, activities and platforms. Based on the commonality of platforms, online courses could be described by the ontology to extract underlying mechanisms and objects. It could also describe patterns of modern education models, such as online education, blended education, micro classroom and flipped classrooms. In addition, it could be applied to computational education science and support innovations of online education strategy.

\section{ACKNOWLEDGMENTS}

This work has been supported by the Jiangsu Modern Education Technology Project 2021-R-87014.

\section{REFERENCES}

[1] RASHID M, RIZZO G, TORCHIANO M, et al. Knowledge Base Evolution Analysis: A Case Study in the Tourism Domain[M/OL]. PAUTASSO C, SÁNCHEZ-FIGUEROA F, SYSTÄ K, et al.//Current Trends in Web Engineering. Cham: Springer International Publishing, 2018: 268-278[2021-04-28].

[2] RASHEED R A, KAMSIN A, ABDULLAH N A. Challenges in the Online Component of Blended Learning: A Systematic Review[J]. Computers \& Education, 2020, 144: 103701.

[3] SCHEMA. Educational Organization[EB/OL](2021). https://schema.org/EducationalOrganization.

[4] CHIMALAKONDA S, NORI K V. An Ontology Based Modeling Framework for Design of Educational Technologies[J]. Smart Learning Environments, 2020, 7(1): 28 .

[5] MALIK S K, PRAKASH N, RIZVI S A M.

Developing a University Ontology in Education Domain Using Protégé for Semantic Web[J]. International Journal of Engineering Science and Technology, 2010, 2: 9

[6] NAHHAS S, BAMASAG O, KHEMAKHEM M, et al. Bridging Education and Labor Skills by a Novel Competency-Based Course Linked-Data Model[J]. IEEE Access, 2019, 7: 119087-119098.

[7] ZHU X. Educational Ontology[EB/OL](2021). https://github.com/aisight/onto-edu. 